\title{
Efficacy and Safety of Favipiravir in Moderate COVID- 19 Pneumonia Patients without Oxygen Therapy: A Randomized, Phase III Clinical Trial
}

\begin{abstract}
Masaharu Shinkai $\cdot$ Kenji Tsushima $\cdot$ Shingo Tanaka $\cdot$ Eri Hagiwara $\cdot$ Norihito Tarumoto $\cdot$ Ichiro Kawada • Yuji Hirai · Sho Fujiwara $\cdot$ Yuko Komase $\cdot$ Takeshi Saraya $\cdot$ Hidefumi Koh · Naho Kagiyama $\cdot$ Megumi Shimada Daiki Kanou · Shinichi Antoku • Yujiro Uchida · Yutaka Tokue • Mikio Takamori · Yasuhiro Gon · Kenya Ie • Yoshitaka Yamazaki Kazumasa Harada - Naoki Miyao · Takashi Naka • Mitsunaga Iwata $\cdot$ Atsushi Nakagawa Kazutoshi Hiyama · Yoshihiko Ogawa · Masahiro Shinoda · Shinichiro Ota · Takatomo Hirouchi · Jiro Terada • Shuichi Kawano · Takashi Ogura • Tsutomu Sakurai • Yoshihiko Matsumoto • Hiroyuki Kunishima • Osamu Kobayashi · Satoshi Iwata
\end{abstract}

Received: May 21, 2021 / Accepted: July 27, 2021 / Published online: August 27, 2021

(C) The Author(s) 2021

\section{ABSTRACT}

Introduction: Severe acute respiratory syndrome coronavirus 2 (SARS-CoV-2), the cause of coronavirus disease 2019 (COVID-19), is an enveloped, single-stranded RNA virus. Favipi-

Supplementary Information The online version contains supplementary material available at https:// doi.org/10.1007/s40121-021-00517-4.

M. Shinkai $\cdot$ M. Shinoda $\cdot$ S. Ota $\cdot$ T. Hirouchi Department of Respiratory Medicine, Tokyo Shinagawa Hospital, 6-3-22 Higashioi, Shinagawaku, Tokyo 140-8522, Japan

K. Tsushima $\cdot$ J. Terada

Department of Pulmonary Medicine, School of Medicine, International University of Health and Welfare, 852 Hatakeda, Narita, Chiba 286-8520, Japan

\section{S. Tanaka}

Department of Otolaryngology, Self-Defense Forces Central Hospital, 1-2-24 Ikejiri, Setagaya-ku, Tokyo 154-8532, Japan

\section{E. Hagiwara · T. Ogura}

Department of Respiratory Medicine, Kanagawa

Cardiovascular and Respiratory Center, 6-16-1

Tomiokahigashi, Kanazawa-ku, Yokohama,

Kanagawa 236-0051, Japan ravir is an orally administrable antiviral drug whose mechanism of action is to selectively inhibit RNA-dependent RNA polymerase. A preliminary trial in COVID-19 patients reported significant improvements across a multitude of clinical parameters, but these findings have not been confirmed in an adequate well-controlled trial. We conducted a randomized, single-blind, placebo-controlled Phase III trial assessing the efficacy and safety of favipiravir in patients with

\section{N. Tarumoto}

Department of Infectious Disease and Infection control, Saitama Medical University Hospital, 38 Morohongo, Moroyama-machi, Iruma-gun, Saitama 350-0495, Japan

\section{Kawada}

Division of Pulmonary Medicine, Department of Medicine, School of Medicine, Keio University, 35 Shinanomachi, Shinjuku-ku, Tokyo 160-8582, Japan

Y. Hirai

Department of Infectious Diseases, Tokyo Medical University Hachioji Medical Center, 1163

Tatemachi, Hachioji, Tokyo 193-0998, Japan

\section{S. Fujiwara}

Department of Infectious Diseases, Tokyo

Metropolitan Cancer and Infectious Diseases Center

Komagome Hospital, 3-18-22 Honkomagome,

Bunkyo-ku, Tokyo 113-8677, Japan 
moderate pneumonia not requiring oxygen therapy.

Methods: COVID-19 patients with moderate pneumonia $\left(\mathrm{SpO}_{2} \geq 94 \%\right)$ within 10 days of onset of fever (temperature $\geq 37.5^{\circ} \mathrm{C}$ ) were assigned to receive either placebo or favipiravir (1800 mg twice a day on Day 1, followed by $800 \mathrm{mg}$ twice a day for up to 13 days) in a ratio of $1: 2$. An adaptive design was used to re-estimate the sample size. The primary endpoint was a composite outcome defined as the time to improvement in temperature, oxygen saturation levels $\left(\mathrm{SpO}_{2}\right)$, and findings on chest imaging, and recovery to SARS-CoV-2-negative. This endpoint was re-examined by the Central Committee under blinded conditions.

Results: A total of 156 patients were randomized. The median time of the primary endpoint was 11.9 days in the favipiravir group and 14.7 days in the placebo group, with a significant difference $(p=0.0136)$. Favipiravir-treated patients with known risk factors such as obesity

\section{Y. Komase}

Department of Respiratory Internal Medicine, St. Marianna University Yokohama City Seibu Hospital, 1197-1 Yasashi-cho, Asahi-ku, Yokohama, Kanagawa 241-0811, Japan

\section{T. Saraya}

Department of Respiratory Medicine, Kyorin University School of Medicine, 6-20-2 Shinkawa, Mitaka, Tokyo 181-8611, Japan

H. Koh

Division of Pulmonary Medicine, Department of Internal Medicine, Tachikawa Hospital, 4-2-22

Nishiki-cho, Tachikawa, Tokyo 190-8531, Japan

N. Kagiyama

Department of Respiratory Medicine, Saitama Cardiovascular and Respiratory Center, 1696 Itai, Kumagaya, Saitama 360-0197, Japan or coexisting conditions provided better effects. Furthermore, patients with early-onset in the favipiravir group showed higher odds ratio. No deaths were documented. Although adverse events in the favipiravir group were predominantly transient, the incidence was significantly higher.

Conclusions: The results suggested favipiravir may be one of options for moderate COVID-19 pneumonia treatment. However, the risk of adverse events, including hyperuricemia, should be carefully considered.

Trial registration: Clinicaltrials.jp number: JapicCTI-205238.

Keywords: COVID-19; Favipiravir; Oral antiviral agent; Phase III clinical trial; Moderate pneumonia not requiring oxygen therapy; SARS-CoV-2; Treatment efficacy
Department of General Internal Medicine, Tokai University Oiso Hospital, 21-1 Gakkyo, Oiso-machi, Naka-gun, Kanagawa 259-0198, Japan

\section{Kanou}

Department of Respiratory Internal Medicine, Yokohama Rosai Hospital, 3211 Kozukue-cho, Kohoku-ku, Yokohama, Kanagawa 222-0036, Japan

\section{S. Antoku}

Department of Diabetes, Metabolism and Kidney Disease, Edogawa Hospital, 2-24-18 Higashikoiwa, Edogawa-ku, Tokyo 133-0052, Japan

Y. Uchida

Department of General Medicine, Kitakyushu Municipal Medical Center, 2-1-1 Bashaku, Kokurakita-ku, Kitakyushu, Fukuoka 802-8561, Japan 


\section{Key Summary Points}

\section{Why carry out this trial?}

Many treatment modalities have been investigated against Coronavirus disease 2019 (COVID-19) which has spread rapidly throughout the world since March 2020; however, there are currently no established antiviral agents that can be orally administered to non-severe cases of COVID-19.

Favipiravir showed the clinical efficacy against COVID-19 in a preliminary trial; however, it has not been confirmed in an adequate well-controlled trial, and it has also not been clarified what characteristics of patients would be suitable for favipiravir.

In a randomized, placebo-controlled trial, we hypothesized that favipiravir would shorten the time to improvement in clinical parameters in moderate COVID19 pneumonia patients not requiring oxygen therapy, with statistical significance.
Y. Tokue

Infection Control and Prevention Center, Gunma University Hospital, 3-39-15 Showamachi,

Maebashi, Gunma 371-8511, Japan

\section{Takamori}

Department of Respiratory Medicine, Tokyo

Metropolitan Tama Medical Center, 2-8-29

Musashidai, Fuchu, Tokyo 183-8524, Japan

Y. Gon

Department of Respiratory Medicine, Nihon University Itabashi Hospital, 30-1 Oyaguchikamicho, Itabashi-ku, Tokyo 173-8610, Japan

K. Ie

Department of General Internal Medicine, Kawasaki Municipal Tama Hospital, 1-30-37 Shukugawara, Tama-ku, Kawasaki, Kanagawa 214-8525, Japan

\section{What was learned from the trial?}

Favipiravir appeared to shortened the time to clinical improvement by approximately 3 days in patients with moderate COVID19 pneumonia presenting with $\mathrm{SpO}_{2} \geq 94 \%$ as per analysis plan. It was also suggested that early administration of favipiravir could be an effective intervention, especially for patients at risk of aggravation. At the same time, adverse events associated with hyperuricemia were observed in more than $80 \%$ of patients. Therefore, the risk of adverse events, including hyperuricemia, needs to be carefully considered when using favipiravir for moderate COVID-19 pneumonia.

\section{INTRODUCTION}

The severe acute respiratory syndrome coronavirus 2 (SARS-CoV-2) that causes coronavirus disease 2019 (COVID-19) has spread rapidly since first described in December 2019. As of May 10, 2021, more than 157,000,000 cases have been reported, with the death toll exceeding 3,200,000 [1]. Under such circumstances, many drugs have been investigated against this emerging viral infection. However, it is hard to say that the therapeutic option for COVID-19 is sufficient.

\section{Y. Yamazaki}

Department of Respiratory and Infectious Diseases, Nagano Prefectural Shinshu Medical Center, 1332

Suzaka, Suzaka, Nagano 382-8577, Japan

\section{K. Harada}

Department of Cardiology, Tokyo Metropolitan Geriatric Hospital and Institute of Gerontology, 352 Sakae-cho, Itabashi-ku, Tokyo 173-0015, Japan

\section{N. Miyao}

Department of Internal Medicine, Nippon Koukan Hospital, 1-2-1 Kokandori, Kawasaki-ku, Kawasaki, Kanagawa 210-0852, Japan

\section{T. Naka}

Department of Neurology, Higashiosaka City Medical Center, 3-4-5 Nishiiwata, Higashiosaka, Osaka 578-8588, Japan 
Favipiravir, approved for use in Japan for novel or re-emerging pandemic influenza virus infections, selectively inhibits viral RNA-dependent RNA polymerase. Favipiravir demonstrates antiviral activity against a broad spectrum of RNA viruses as well as influenza viruses [2, 3]. The drug's inhibitory effects against SARS-CoV-2 were first reported in an in vitro study [4]. Previous reports suggested favipiravir may exert its antiviral activity against SARS-CoV-2 through a combination of chain termination, retarding RNA synthesis, and inducing lethal mutagenesis $[5,6]$. The clinical efficacy of favipiravir was first reported by Cai et al. They reported significant efficacy of favipiravir in the time to SARS-CoV-2 clearance and the time to chest imaging findings improvement compared to lopinavir/ritonavir [7]; however, these findings were obtained by a non-randomized and unblinded design. Thus, the clinical efficacy of favipiravir has not been confirmed in a well-controlled trial. In addition to this issue, it has also been unclear what characteristics of patients would be suitable for favipiravir treatment. We decided to confirm the clinical efficacy of favipiravir in a randomized, placebo-controlled trial, and to find suitable characteristics of patients with COVID-19 for favipiravir treatment.

M. Iwata

Department of Emergency and General Internal Medicine, Fujita Health University Hospital, 1-98 Dengakugakubo, Kutsukake-cho, Toyoake, Aichi 4701192, Japan

\footnotetext{
A. Nakagawa

Department of Respiratory Medicine, Kobe City

Medical Center General Hospital, 2-1-1

Minatojimaminamimachi, Chuo-ku, Kobe, Hyogo

650-0047, Japan

K. Hiyama

Department of Infectious Diseases, National Hospital Organization Fukuokahigashi Medical Center, 1-1-1 Chidori, Koga, Fukuoka 811-3195, Japan

Y. Ogawa

Department of Infectious Diseases, Sakai City

Medical Center, 1-1-1 Ebarajicho, Nishi-ku, Sakai,

Osaka 593-8304, Japan
}

\section{METHODS}

\section{Trial Design and Oversight}

A randomized, single-blind, placebo-controlled, parallel group comparison design was adopted to assess the safety and effectiveness of favipiravir in COVID-19 patients. Due to limited knowledge regarding this treatment at the time of planning, an adaptive design was incorporated to re-estimate the sample size based on the findings from an interim analysis. The observational study for compassionate use of favipiravir for COVID-19 had already been initiated in Japan at the time of this trial [8]. Therefore, many of COVID-19 patients were able to access favipiravir treatment. In order to minimize any disadvantages to patients assigned to the placebo group, the assignment ratio was set at 2:1 in favor of the favipiravir group. Investigators were permitted to switch patients to rescue treatments in the event of "lack of efficacy", defined as marked deterioration in patients' chest images and a continuous downward trend in oxygen saturation levels $\left(\mathrm{SpO}_{2}\right)$ during the $12 \mathrm{~h}$ before and after imaging. In these cases, late administration of favipiravir was permitted as a treatment option for patients in the placebo group. Considering the above, a placebo-controlled trial was considered ethically permissible. This trial plan was filed to Japanese

\section{S. Kawano}

Department of Internal Medicine, Self-Defense Forces Central Hospital, 1-2-24 Ikejiri, Setagaya-ku, Tokyo 154-8532, Japan

T. Sakurai · Y. Matsumoto

Scientific Information Department, FujiFilm

Toyama Chemical Co., Ltd., 3-4-8 Hatchobori, Chuo-ku, Tokyo 104-0032, Japan

H. Kunishima

Department of Infectious Diseases, School of Medicine, St. Marianna University, 2-16-1 Sugao, Miyamae-ku, Kawasaki, Kanagawa 216-8511, Japan

O. Kobayashi $\cdot$ S. Iwata $(\bowtie)$

Department of Infectious Diseases, National Cancer Center Hospital, 5-1-1 Tsukiji, Chuo-ku, Tokyo 1040045, Japan

e-mail: siwata@ncc.go.jp 
regulatory authorities (IND \#2019-7269). The protocol, informed consent form, and all other required documents were reviewed by an Institutional Review Board (IRB) of each trial site prior to initiation of the trial at that site. The trial was approved by all IRBs (please see supplementary material), and was conducted in accordance with the Helsinki Declaration (1964 and its later amendments) and Good Clinical Practice.

\section{Participants}

Since favipiravir is an oral antiviral agent, it is unsuitable for patients with dysphagia such as patients requiring oxygen therapy or with a disturbance of consciousness. Patients with moderate illness requiring inpatient treatment were therefore targeted for this trial. The inclusion criteria were: (1) male or female aged 20-74 years; (2) positive for SARS-CoV-2 based on a nucleic acid amplification test of a respiratory tract sample taken at enrollment, pulmonary lesions confirmed by chest imaging, and fever $\geq 37.5^{\circ} \mathrm{C}$; and (3) written, informed consent obtained from the patient. The main exclusion criteria were: (1) 11 or more days since onset of fever of $\geq 37.5^{\circ} \mathrm{C}$; (2) the infection episode was a relapse or reinfection; (3) $\mathrm{SpO}_{2}<94 \%$ without oxygen therapy. Further details were described in the protocol attached in the electronic supplementary material.

\section{Setting}

Investigators disclosed information regarding the allocated drugs to the enrolled patient only to the minimal necessary stakeholders of the trial site in order to minimize bias. The information on the allocated drugs was not disclosed to the patients or to the Central Committee, and the blinding was maintained throughout the trial. Investigators observed and recorded patient's clinical symptoms and vital signs at least twice a day (morning and evening) until the time of discharge and/or Day 28. Virological examination of SARS-CoV-2 using nasopharyngeal specimens and chest $\mathrm{X}$-rays were performed every 3 days until the time of discharge and/or
Day 28. Chest X-rays and SARS-CoV-2 qualitative tests were able to perform arbitrarily when the investigator evaluated the primary endpoints. Investigators submitted the chest X-rays used for evaluation to the Central Committee. Viral samples for qualitative tests were evaluated at each trial site by the method recommended by the National Institute of Infectious Diseases.

\section{Interventions}

After confirming trial eligibility, central randomization was conducted. Patients assigned to the favipiravir group received favipiravir at $1800 \mathrm{mg}$ per dose twice a day on Day 1 followed by $800 \mathrm{mg}$ per dose twice a day from Day 2 for up to 13 days. This dosage was higher than the approved dosage in Japan for influenza. This is because the $\mathrm{EC}_{50}$ of favipiravir for the influenza A (H5N1) virus by neutral red uptake assay ranged from $0.4-1.9 \mu \mathrm{g} / \mathrm{mL}$ [9], while the $\mathrm{EC}_{50}$, for SARS-CoV-2 was $9.72 \mu \mathrm{g} / \mathrm{mL}$ [4], which was higher. It has been reported that when the same favipiravir dose as in the present trial was administered to Japanese patients with severe fever with thrombocytopenia syndrome (SFTS), the mean trough concentration reached $40 \mu \mathrm{g} /$ $\mathrm{mL}$ [10]. Therefore, this dosage was adopted in the trial. Patients assigned to the placebo group received matching placebo tablets for up to 14 days. Inpatient management was mandatory throughout the duration of study drug treatment. The use of interferon alpha, any drug reported to have an antiviral effect against SARS-CoV-2 (remdesivir, hydroxychloroquine sulfate, chloroquine phosphate, lopinavir/ritonavir, ciclesonide, nafamostat mesilate, camostat mesilate, nelfinavir mesilate, and ivermectin), and any type of hemofiltration therapy was prohibited.

\section{Outcomes}

At the time of this trial, the Japanese regulatory authorities stipulated that viral qualitative tests by nucleic acid amplification could be performed the day after improvement in fever and respiratory symptoms, and that, if negative 
results were obtained for two consecutive days, COVID-19 patients could be discharged. With reference to this criterion, the primary endpoint was a composite outcome defined as the time to improvement in four clinical parameters (temperature, $\mathrm{SpO}_{2}$, findings on chest imaging, and viral clearance). SARS-CoV-2 qualitative testing using nucleic acid amplification of samples obtained at least $24 \mathrm{~h}$ after improvement in the other clinical parameters (temperature, $\mathrm{SpO}_{2}$, and findings on chest imaging) were documented. Improvement was defined as follows: (1) improvement in temperature was defined as axillary temperature falling to $\leq 37.4{ }^{\circ} \mathrm{C}$ and remaining at $\leq 37.4{ }^{\circ} \mathrm{C}$ for at least $24 \mathrm{~h}$ (temperature recordings taken within $4 \mathrm{~h}$ after use of an antipyretic were excluded); (2) improvement in $\mathrm{SpO}_{2}$ was defined as $\mathrm{SpO}_{2}$ remaining $\geq 96 \%$ for at least $24 \mathrm{~h}$ without the use of oxygen therapy; (3) improvement on chest imaging was defined as improvement in chest imaging findings taken at least $24 \mathrm{~h}$ after the previous image judged to be the worst; and (4) recovery to SARS-CoV-2-negative was defined as two consecutive negative results on qualitative tests by nucleic acid amplification separated by at least $24 \mathrm{~h}$. If the above definition was met, the time to become negative for the first time in a qualitative test was defined as the improvement time in the primary endpoint. In other words, if all four clinical parameters were alleviated, the patient was judged to be able to be discharged.

In addition to the primary endpoint, several secondary endpoints were also evaluated. Regarding safety assessment, all adverse events (AEs) which occurred during 28 days and reported by investigators were tabulated.

\section{Sample Size}

Based on the preceding reports [7], it was estimated that the time to symptom improvement would be 4 days shorter in the favipiravir group. Assuming that the survival function of days to improvement follows an exponential distribution, it was determined that 96 patients (64 favipiravir, 32 placebo) would be sufficient to demonstrate the statistical superiority of favipiravir by the log-rank test with $\alpha=0.05$ (two-sided) and statistical power of $80 \%$. The protocol stipulated that the sample size could be re-estimated based on the results of an interim analysis by the Central Committee. The interim analysis was held when data from $\geq 45$ patients had been obtained, as stipulated in the protocol. The Committee biostatistician recommended that a sample size of at least 144 patients (96 favipiravir, 48 placebo) be adopted, assuming a study dropout rate of $10 \%$ and patients disease recovery rate before treatment initiation of $25 \%$. The final target sample size was therefore set to 144 patients (96 favipiravir, 48 placebo).

\section{Independent Central Committee}

Operational bias associated with a single-blind design was minimized by results being reassessed by the Central Committee under blinded conditions. Regarding the re-assessment of chest imaging findings, the Committee identified the worst images from the extent and density of the lesions on chest X-ray images submitted by the investigator with the consensus of all members. In the event of a discrepancy between the Committee and the investigator, the opinion of the Committee was adopted. The Committee members included five external experts: four physicians including a radiologist, and one biostatistician. The Committee was assigned three duties: (1) re-assessing the primary endpoint; (2) assessing the validity of a judgment on the lack of efficacy; and (3) re-estimating the target sample size. To minimize evaluation bias, re-estimated sample-size was not disclosed to all investigators and the Committee members other than the biostatistician. The Committee gathering was held three times. Upon re-assessing the primary endpoint, the Committee members were not provided with data other than body temperature, $\mathrm{SpO}_{2}$, SARSCoV-2 qualitative tests, and chest imaging findings related to the primary endpoint.

\section{Statistical Analysis}

For the analysis of the primary endpoint, the log-rank test based on the weighted $Z$ statistic 


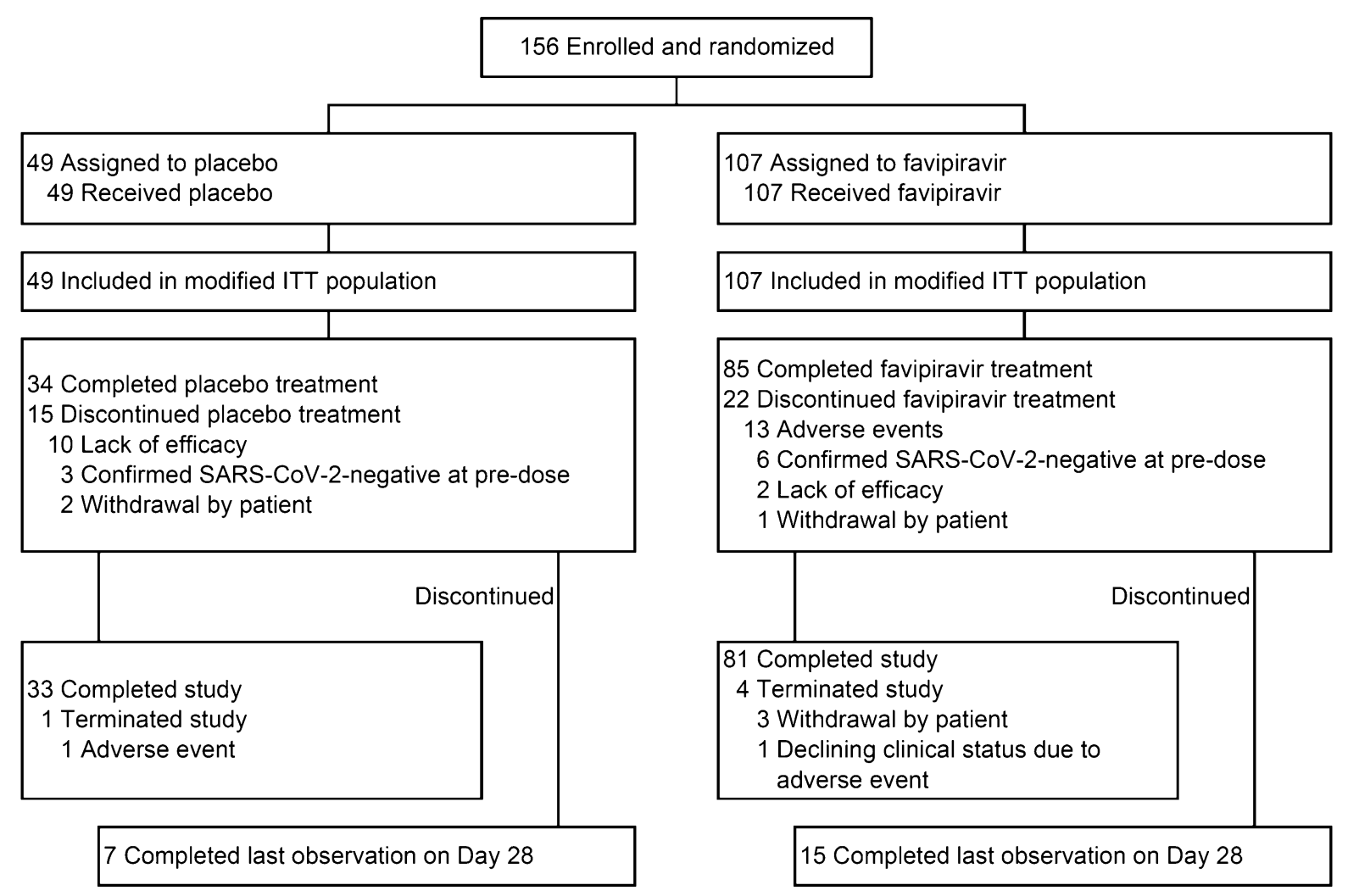

Fig. 1 Of the 156 patients who underwent randomization, 119 (85 favipiravir, 34 placebo) completed the assigned study drug treatment. The study drug was discontinued in 22 patients in the favipiravir group and 15 in the placebo group, and, among these patients, the scheduled observations were continued to Day 28 in 15

[11] was used to control the Type 1 error before and after the interim analysis. Hazard ratios (HRs) and their 95\% confidence intervals (CIs) were also calculated using the Cox proportional hazards model with sex and age at baseline as covariates. Lack of efficacy cases were censored on Day 28. Longitudinal data such as the patient status were assessed using a mixed-effects model for repeated measures. The differences in least square means between treatments and the 95\% CIs were then calculated and compared. SAS software v.9.4 (SAS Institute, Cary, NC, USA) was used for these analyses. All statistical tests were two-tailed. $p$ values less than 0.05 were considered significant.

patients in the favipiravir group and 7 in the placebo group. The study was discontinued for 4 patients in the favipiravir group and 1 in the placebo group. All 156 patients (107 favipiravir, 49 placebo) were included in the efficacy analysis as modified ITT population

\section{RESULTS}

\section{Patients}

A total of 156 patients were randomized (Fig. 1). All patients who received the study drug were included in the analyses. Eighty-five patients in the favipiravir group and 34 patients in the placebo group completed the study drug treatment, of which 81 patients in the favipiravir group and 33 patients in the placebo group completed the study. The investigator documented a lack of efficacy in 2 patients in the favipiravir group and 10 patients in the placebo group; however, the Central Committee overruled the judgment of a lack of efficacy in 1 patient in the placebo group. Seven patients in the placebo group were switched to treatment 
Table 1 Patients' baseline demographic and clinical characteristics

\begin{tabular}{|c|c|c|}
\hline Characteristic & $\begin{array}{l}\text { Placebo } \\
(n=49)\end{array}$ & $\begin{array}{l}\text { Favipiravir } \\
(n=107)\end{array}$ \\
\hline \multicolumn{3}{|l|}{ Sex } \\
\hline Male & $28(57.1 \%)$ & $76(71.0 \%)$ \\
\hline Female & $21(42.9 \%)$ & $31(29.0 \%)$ \\
\hline \multicolumn{3}{|l|}{ Age (years) } \\
\hline Mean (SD) & $48.7(14.1)$ & $43.8(12.5)$ \\
\hline$<65$ & $42(85.7 \%)$ & $101(94.4 \%)$ \\
\hline$\geq 65$ & $7(14.3 \%)$ & $6(5.6 \%)$ \\
\hline \multicolumn{3}{|l|}{ Weight (kg) } \\
\hline Mean $(\mathrm{SD})$ & $69.7(13.8)$ & $73.2(15.8)$ \\
\hline \multicolumn{3}{|l|}{ BMI $\left(\mathrm{kg} / \mathrm{m}^{2}\right)$} \\
\hline Mean (SD) & $25.3(4.1)$ & $25.5(4.4)$ \\
\hline \multicolumn{3}{|l|}{$\mathrm{CL}_{\mathrm{cr}}(\mathrm{mL} / \mathrm{min})$} \\
\hline Mean (SD) & $108.4(29.1)$ & $118.1(34.3)$ \\
\hline \multicolumn{3}{|l|}{ Race } \\
\hline Asian & $49(100 \%)$ & $107(100 \%)$ \\
\hline Other & $0(0 \%)$ & $0(0 \%)$ \\
\hline \multicolumn{3}{|c|}{ Coexisting conditions $^{\mathrm{a}}$} \\
\hline No & $35(71.4 \%)$ & $83(77.6 \%)$ \\
\hline Yes & $14(28.6 \%)$ & $24(22.4 \%)$ \\
\hline \multicolumn{3}{|c|}{ Time from onset of fever above $37.5^{\circ} \mathrm{C}$ (days) } \\
\hline Mean (SD) & $5.7(2.8)$ & $4.8(2.3)$ \\
\hline \multicolumn{3}{|c|}{ Temperature $\left({ }^{\circ} \mathrm{C}\right)$} \\
\hline Mean $(S D)$ & $37.7(0.8)$ & $37.8(0.9)$ \\
\hline \multicolumn{3}{|c|}{ Respiratory rate $(/ \mathrm{min})$} \\
\hline Mean $(S D)$ & $18.6(3.3)$ & $19.3(3.9)$ \\
\hline \multicolumn{3}{|l|}{$\mathrm{SpO}_{2}(\%)$} \\
\hline Mean (SD) & $96.0(2.1)$ & $96.1(1.7)$ \\
\hline \multicolumn{3}{|c|}{ Viral load $\left(\log _{10} \text { copies } / \mathrm{mL}\right)^{\mathrm{b}}$} \\
\hline Mean (SD) & $4.82(1.92)$ & $5.00(1.98)$ \\
\hline \multicolumn{3}{|l|}{$\mathrm{NEWS}^{\mathrm{c}}$} \\
\hline Mean (SD) & $1.9(1.8)$ & $2.3(2.0)$ \\
\hline
\end{tabular}

Table 1 continued

\begin{tabular}{lll}
\hline Characteristic & $\begin{array}{l}\text { Placebo } \\
(\boldsymbol{n}=49)\end{array}$ & $\begin{array}{l}\text { Favipiravir } \\
(\boldsymbol{n}=\mathbf{1 0 7})\end{array}$ \\
\hline $\begin{array}{ll}\text { Clinical risk based on NEWS } \\
\text { High }\end{array}$ & $1(2.0 \%)$ & $4(3.7 \%)$ \\
Medium & $4(8.2 \%)$ & $19(17.8 \%)$ \\
Low & $43(87.8 \%)$ & $83(77.6 \%)$ \\
Unknown & $1(2.0 \%)$ & $1(0.9 \%)$ \\
Clinical status score & \\
1 & $0(0 \%)$ & $0(0 \%)$ \\
2 & $0(0 \%)$ & $0(0 \%)$ \\
3 & $48(98.0 \%)$ & $106(99.1 \%)$ \\
4 & $1(2.0 \%)$ & $1(0.9 \%)$ \\
5 & $0(0 \%)$ & $0(0 \%)$ \\
6 & $0(0 \%)$ & $0(0 \%)$ \\
7 & $0(0 \%)$ & $0(0 \%)$ \\
\hline
\end{tabular}

${ }^{a}$ Coexisting conditions included prognostic factors such as chronic respiratory illness, chronic renal disease, diabetes mellitus, hypertension, and cardiovascular illness

b SARS-CoV-2 genome load in nasopharyngeal swabs

c National Early Warning Score

d Low was defined as a total score of 0 to 4 points on NEWS; Medium was defined as a total score of 5 to 6 points on NEWS or at least 3 points for one of the parameters that make up NEWS; High was defined as a total score of 7 points or more on NEWS

e 7-point ordinary scale: 1 not hospitalized, no limitations on activities; 2 not hospitalized, limitation on activities; 3 hospitalized, not requiring supplemental oxygen; 4 hospitalized, requiring supplemental oxygen; 5 hospitalized, on non-invasive ventilation or high-flow oxygen devices; 6 hospitalized, on invasive mechanical ventilation or extracorporeal membrane oxygenation; 7 death

with favipiravir during Days 2-8 due to a lack of efficacy. Of the 156 patients, the proportion of males assigned to the favipiravir was relatively higher. Mean age did not differ between the two groups, but the proportion of patients aged $\geq 65$ years was higher in the placebo group. Regarding NEWS, the proportion of high/ 
medium-risk patients was higher in the favipiravir group. The two groups were otherwise well balanced (Table 1). The mean $\mathrm{SpO}_{2}$ prior to treatment initiation was $96 \%$ and more in both groups, as patients with COVID-19 pneumonia not requiring oxygen therapy were included.

\section{Efficacy}

The median time (95\% CI) to meet the primary endpoint was 11.9 (10.0-13.1) days in the favipiravir group and 14.7 (10.5-17.9) days in the placebo group (Fig. 2a; Table 2). Patients receiving favipiravir showed median values of approximately 3 days shorter, with a significant difference $(p=0.0136)$. In the covariate-adjusted Cox proportional hazards model, the HR (95\% CI) was 1.59 (1.02-2.48), which was also significant. The primary endpoint showed significant differences in both methods.

Regarding the individual parameters constituting the primary endpoint, the median time to improvement of chest imaging findings was significantly shorter in the favipiravir group than in the placebo group $(p=0.0287)$. The median time to recovery to SARS-CoV-2-negative on qualitative testing was also significantly shorter in the favipiravir group ( $p=0.0405)$, but those of the remaining two parameters (temperature and $\mathrm{SpO}_{2}$ ) were comparable (Table 2).

Of the individual COVID-19 symptoms, headache, myalgia or arthralgia, and fatigue or tiredness improved significantly earlier in the favipiravir group (Fig. 3). Cyanosis, pleural effusion and disorientation could not be fully investigated because the number of documented cases was fewer. The difference in the cumulative chest imaging improvement rates became increasingly apparent over time, and, from Day 10, a significant difference was noted (Fig. 4).

With respect to patient status using the 7-point ordinal scale, the difference in the proportion of patients discharged by Day 21 was $6.7 \%$, with no significant difference between the groups (Fig. 5).

In the predefined sub-group analyses, lymphocyte subsets, LDH, and CRP were included as laboratory test parameters, based on a report of the characteristics of Japanese COVID-19 patients with pneumonia [12]. The each of the sub-groups of patients with BMI of $\geq 30 \mathrm{~kg} / \mathrm{m}^{2}$, $<5$ days from onset, high viral genome load, high CRP, low lymphocytes, and coexisting conditions (chronic respiratory illness, chronic renal disease, diabetes mellitus, hypertension, and cardiovascular illness) had a significantly higher HR (Fig. 6).

\section{Ad Hoc Analysis}

IgA and IgG antibodies were measured, and subgroup analyses were performed. In the favipiravir group, the median time to meet the primary endpoint was 2.9 days shorter in the IgA or IgG antibody-positive sub-group (Fig. 2b). In the IgA and IgG antibody-negative sub-group, there was a significant difference between the two treatment groups by the log-rank test $(p=0.0024)$. The covariate-adjusted HR $(95 \%$ CI) based on the Cox proportional hazards model was 2.07 (1.19-3.60), which was also significantly higher (Fig. 2c). A similar trend was noted in patients who were negative for IgA or IgG antibodies.

\section{Safety}

No deaths were documented in the present trial. The incidence of AEs was 106 (93.0\%) of 114 patients in the favipiravir group, including patients who switched from placebo to favipiravir, and 19 (38.8\%) of 49 patients in the placebo group, with a significant difference between the groups. Four serious adverse events (SAEs) were documented in the favipiravir group (Table 3). SAEs in the favipiravir group were cardiopulmonary arrest, cerebral infarction, liver disorder, and COVID-19-related pneumonia. Of these SAEs, a causal relationship could not be ruled out were cerebral infarction and liver disorder. The incidence of AEs for which the outcome was improvement or recovery was comparable, and the incidence of AEs that did not require intervention was significantly higher in the favipiravir group at 177 (65.6\%) of 270 events compared to 10 (31.3\%) of 32 events in the placebo group, respectively. 

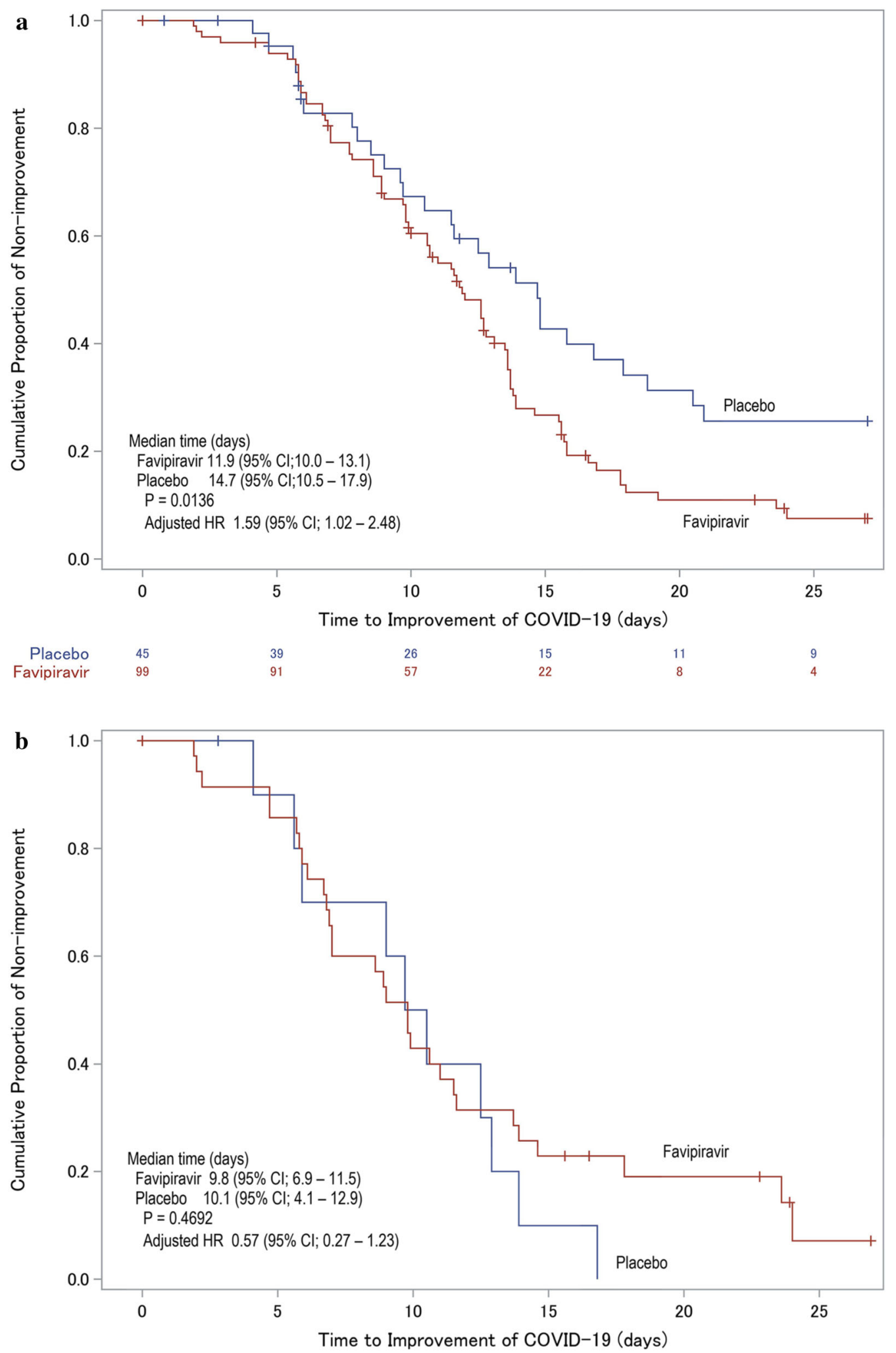

\begin{tabular}{|c|c|c|c|c|c|}
\hline Placebo & 12 & 9 & 5 & 1 & 0 \\
\hline Favipiravir & 36 & 30 & 15 & 8 & 5 \\
\hline
\end{tabular}


4Fig. 2 Kaplan-Meier curves for improvement in COVID19 symptoms (i.e., temperature, $\mathrm{SpO} 2$, and findings on chest imaging, and SARS-CoV-2-negative conversion on qualitative testing) for 28 days defined as the primary end point among all patients in the trial (primary analysis) (Panel a) and two sub-groups according to whether the patients had IgA or IgG antibodies (Panel b) or not (Panel c) at randomization. The red curve represents the favipiravir group, and the blue curve represents the placebo group. The $p$ value for the primary analysis (Panel a) was calculated by the log-rank test based on the weighted $Z$ statistic. The others (Panels $\mathbf{b}$ and $\mathbf{c}$ ) were calculated by a log-rank test. Hazard ratios (HR) for favipiravir to placebo were calculated using the Cox proportional hazards model adjusted for sex and age at baseline
(9/114), ALT increased 7.0\% (8/114), and hepatic function abnormal 5.3\% (6/114). Favipiravir-specific AEs associated with hyperuricemia occurred in 87 (76.3\%) of 114 patients (see Table S1 in the electronic supplementary material for details).

\section{DISCUSSION}

Favipiravir has so far shown clinical efficacy against COVID-19 in several unblinded trials and has become a treatment option in China, India, and Russia [7, 13, 14]. However, some unidentified findings still exist. We conducted a randomized, single-blind, placebo-controlled

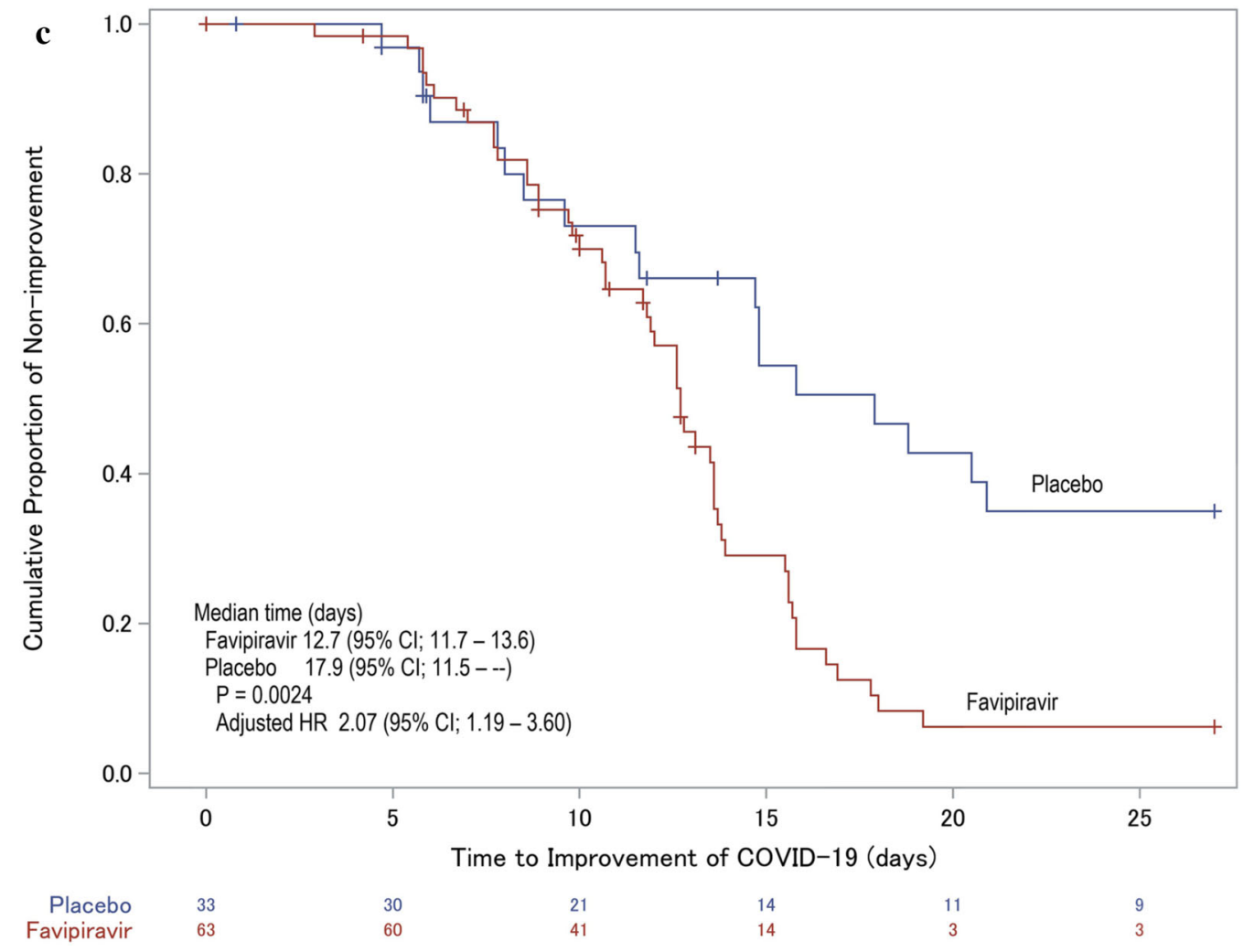

Fig. 2 continued

AEs in the favipiravir group with $\geq 5 \%$ incidence were hyperuricemia $38.6 \% \quad(44 / 114)$, increased blood uric acid $37.7 \%$ (43/114), pyrexia and increased hepatic enzymes both $7.9 \%$
Phase III trial to confirm that favipiravir significantly shortens the time to improvement in clinical parameters in moderate COVID-19 pneumonia patients without oxygen therapy. 
Table 2 Time from study drug initiation to COVID-19 clinical parameter improvement

Placebo $(n=49)$

Favipiravir $(n=107)$

Primary endpoint

Number of patients who improved

28

21

81

Number of patients censored

$75 \%$ time point of improvement (days) (95\% CI)

Median time to improvement (days) (95\% CI)

$25 \%$ time point of improvement (days) (95\% CI)

$p$ value (log-rank test based on weighted $Z$ statistic)

Adjusted hazard ratio (Favipiravir vs. placebo)

(95\% CI)

$-$

$14.7(10.5-17.9)$

$9.0(5.8-11.6)$

26

$15.6(13.7-17.8)$

$11.9(10.0-13.1)$

$7.8(6.7-9.7)$

0.0136

1.59

(1.02-2.48)

Temperature

Number of patients who improved

30

70

Number of patients censored

19

37

Median time to improvement (days) (95\% CI)

$2.1(1.0-3.7)$

$2.0(1.5-3.1)$

$p$ value (log-rank test)

0.1760

Adjusted hazard ratio (Favipiravir vs. placebo)

(95\% CI)

1.36

$(0.87-2.13)$

$\mathrm{SpO}_{2}$

Number of patients who improved

26

48

Number of patients censored

23

59

Median time to improvement (days) (95\% CI)

$2.7(2.3-5.0)$

$2.9(1.8-4.7)$

$p$ value (log-rank test)

0.5110

Adjusted hazard ratio (Favipiravir vs. placebo)

(95\% CI)

1.37

$(0.83-2.25)$

Chest imaging

Number of patients who improved

35

95

Number of patients censored

14

12

Median time to improvement (days) (95\% CI)

$5.7(2.9-6.0)$

$4.8(3.1-5.7)$

$p$ value (log-rank test)

0.0287

Adjusted hazard ratio (Favipiravir vs. placebo)

1.43

(95\% CI)

(0.95-2.15)

SARS-CoV-2 (qualitative)

Number of patients with undetectable SARS-CoV-2 
Table 2 continued

\begin{tabular}{lll}
\hline & Placebo $(\boldsymbol{n}=49)$ & Favipiravir $(\boldsymbol{n}=\mathbf{1 0 7})$ \\
\hline Number of patients censored & 18 & 20 \\
Median time to recovery (days) $(95 \% \mathrm{CI})$ & $12.1(8.5-14.8)$ & $11.0(9.7-12.6)$ \\
$p$ value (log-rank test) & 0.0405 \\
Adjusted hazard ratio (Favipiravir vs. placebo) & 1.40 \\
$(95 \% \mathrm{CI})$ & $(0.91-2.14)$ \\
\hline
\end{tabular}

The primary endpoint was defined as the time from study drug initiation to improvement in temperature, $\mathrm{SpO}_{2}$, and findings on chest imaging, and recovery to SARS-CoV-2-negative on qualitative testing using nucleic acid amplification from at least $24 \mathrm{~h}$ after improvement. The $p$ value for the primary endpoint was calculated by the log-rank test based on the weighted $Z$ statistic to correct for Type 1 errors before and after the interim analysis. The other $p$ values were calculated by a log-rank test. Hazard ratios for favipiravir to placebo were calculated using the Cox proportional hazards model adjusted for sex and age at baseline

Significance was examined by the confidence interval method. The lower limit of the $95 \%$ confidence interval for HR is greater than 1.0, which means that favipiravir is significantly faster than placebo ( $\mathrm{HR}=1$ means equivalent)

The hypothesis of the present trial was established with reference to a previous trial conducted by Cai et al. [7], and although the median values were slightly longer than anticipated, the difference of approximately 3 days was not inconsistent with the hypothesis. The primary endpoint of this trial could be viewed as readily influenced by the timing of evaluation or frequency of chest imaging and virus qualitative tests that can be measured arbitrarily by the investigators. However, no clear differences were noted between the two groups regarding these evaluation timing and frequencies (see Table S2 in the electronic supplementary material for details). In addition, our results were evaluated under blind by an independent Central Committee in a manner equivalent to a double-blind study. Collectively, the efficacy of favipiravir was thought to have been confirmed with reliability. Since there

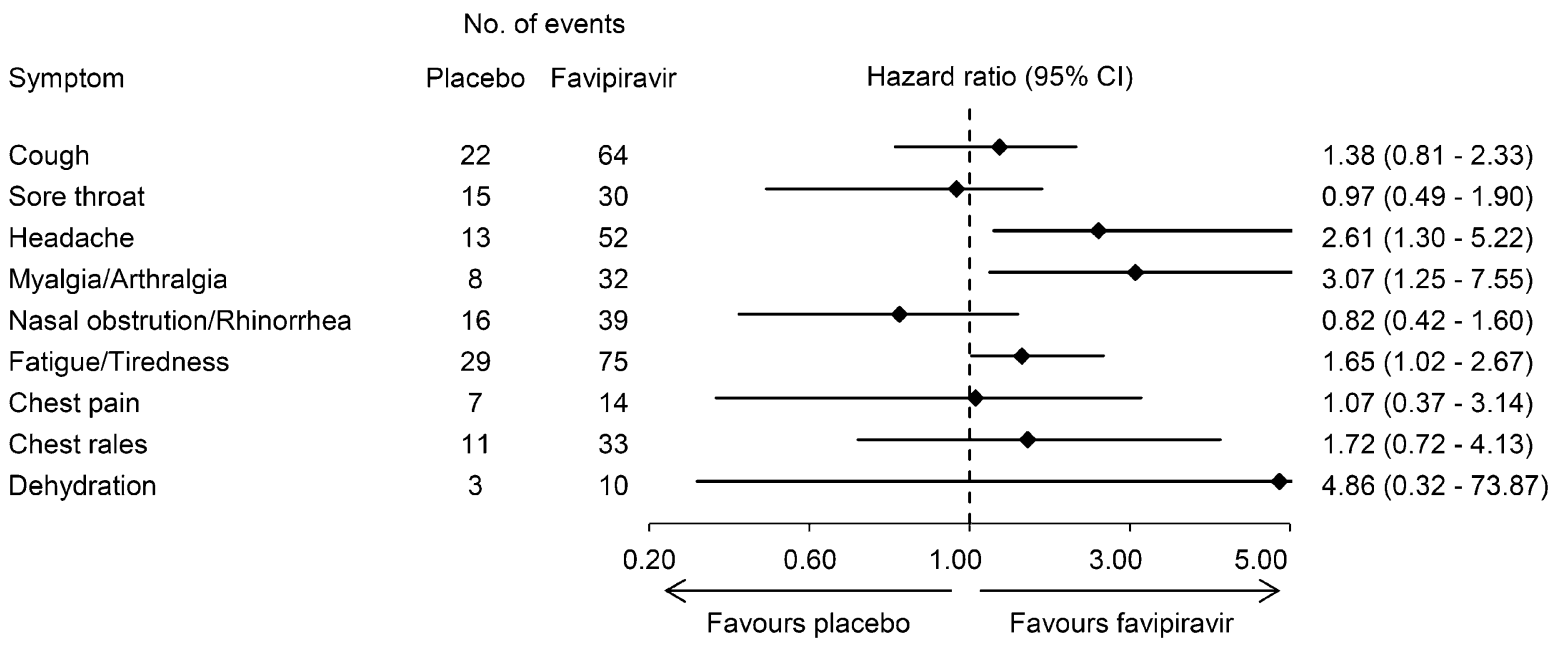

Fig. 3 Individual symptom specific hazard ratios using the Cox proportional hazards model adjusted for sex, age, the categorical variables that correspond to individual symptoms, and their interactions. Hazard ratios are plotted as black diamonds. Horizontal lines represent 95\% confidence intervals 


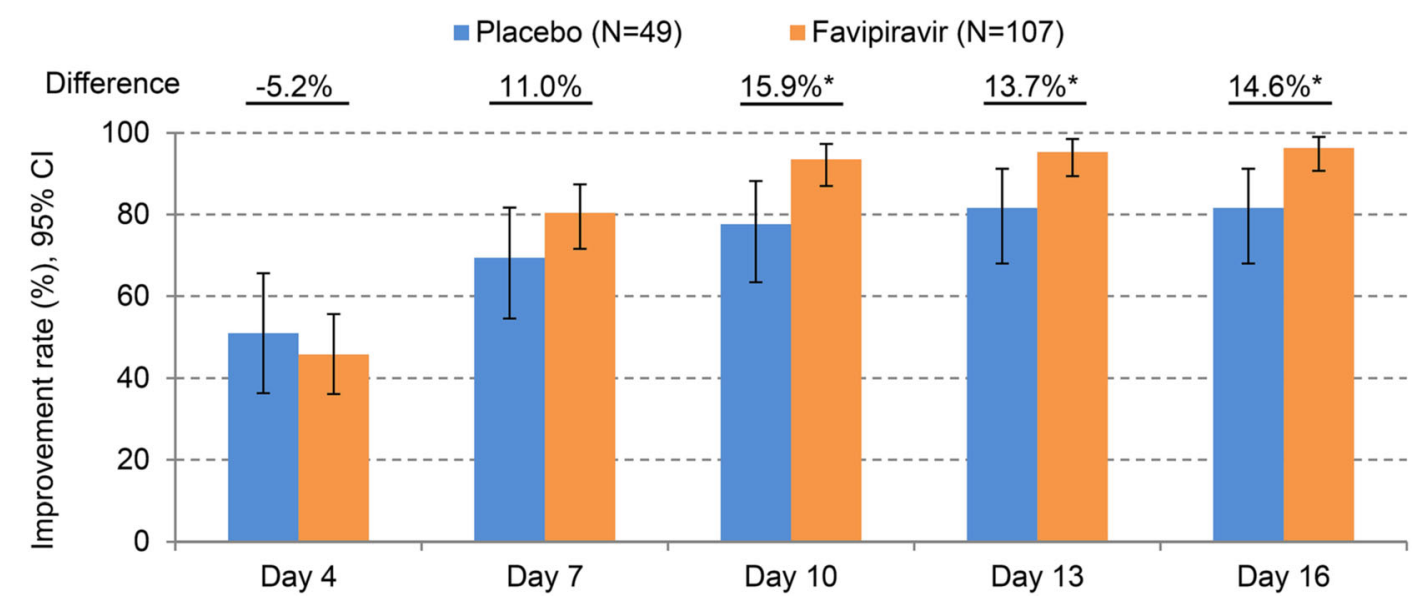

Fig. 4 Time course of cumulative improvement rates on chest X-ray imaging. An asterisk on the difference in improvement rate indicates that there is a significant difference

were imbalances in the distribution of patient age and NEWS at baseline, the effect was evaluated using a Cox proportional hazards model with these as covariates. The HR (95\% CI) adjusted with these covariates was 1.68 (1.06-2.64), which was above the original HR. From this analysis, these imbalances were not considered to be impacts sufficient to bias the results. A sensitivity analysis when events after discontinuation were regarded as effects of the assigned study drug was also conducted to determine the impact of a lack of efficacy, including 7 patients who were switched to favipiravir treatment in the placebo group. This analysis showed the time to improvement in the favipiravir group was approximately 2 days shorter, confirming the same trends as the primary analysis. Regarding the clinical parameters constituting the primary endpoint, there were no significant differences in the time to improvement of temperature and $\mathrm{SpO}_{2}$ when assessed as standalone factors. Interestingly,

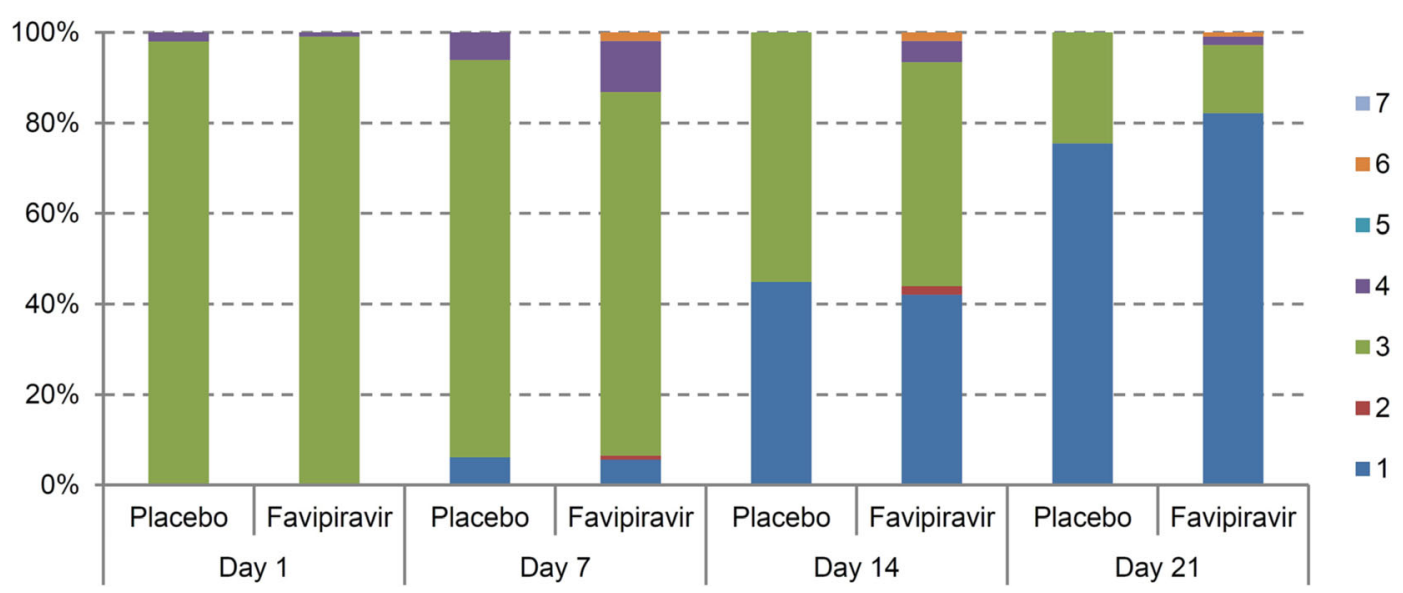

Fig. 5 Time course of patient status in accordance with the 7-point ordinal scale. Any missing clinical status score after the day of discharge was imputed by status score 1 (not hospitalized, no limitations on activities); other missing scores were imputed by last observation carried forward. Each number means the following clinical status; 1 not hospitalized, no limitations on activities; 2 not hospitalized, limitation on activities; 3 hospitalized, not requiring supplemental oxygen; 4 hospitalized, requiring supplemental oxygen; 5 hospitalized, on non-invasive ventilation or high-flow oxygen devices; 6 hospitalized, on invasive mechanical ventilation or extracorporeal membrane oxygenation; 7 death 
No. of patients

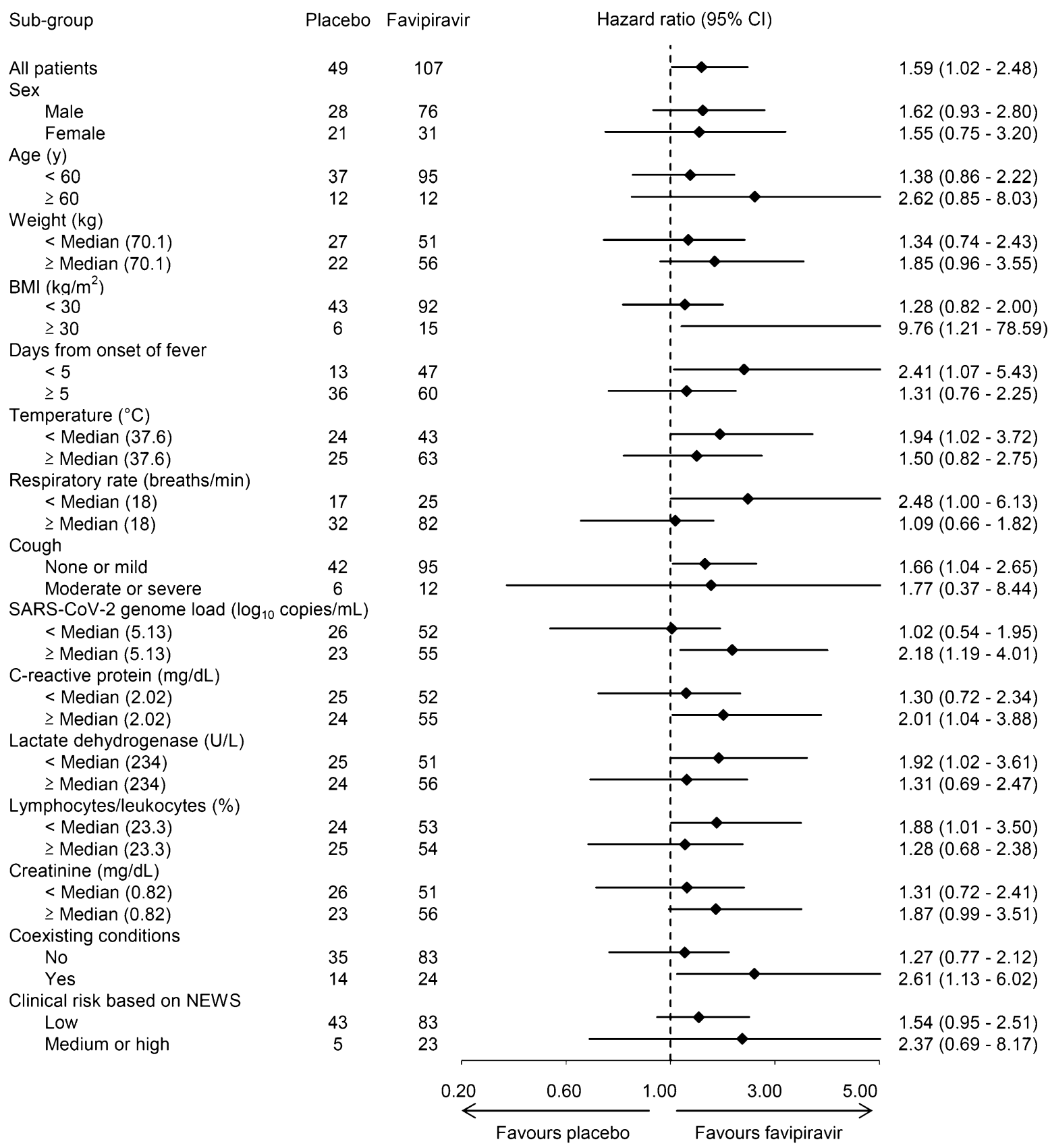

Fig. 6 Sub-group specific hazard ratios using the Cox proportional hazards model adjusted for sex, age, the categorical variables that correspond to sub-groups, and their interactions. Hazard ratios are plotted as black diamonds. Horizontal lines represent $95 \%$ confidence ntervals. Nine and five patients in the favipiravir and placebo groups, respectively, were censored on Day 0 because they were SARS-CoV-2-negative before treatment initiation. Coexisting conditions included prognostic factors such as chronic respiratory illness, chronic renal disease, diabetes mellitus, hypertension, and cardiovascular illness 
Table 3 Adverse event summary

\begin{tabular}{|c|c|c|c|}
\hline Adverse event (AE) & $\begin{array}{l}\text { Placebo } \\
(N=49)\end{array}$ & $\begin{array}{l}\text { Favipiravir } \\
(N=107)^{\mathrm{a}}\end{array}$ & $\begin{array}{l}\text { Pooled favipiravir } \\
(N=114)^{\mathbf{b}}\end{array}$ \\
\hline \multicolumn{4}{|l|}{ Any AE } \\
\hline No. of patients with $\mathrm{AE}$ & 19 & 99 & 106 \\
\hline No. of AEs & 32 & 254 & 270 \\
\hline Incidence $\%$ & 38.8 & 92.5 & 93.0 \\
\hline $95 \%$ confidence interval $^{c}$ & $25.2-53.8$ & $85.8-96.7$ & $86.6-96.9$ \\
\hline Difference (vs. Placebo) & & 53.7 & 54.2 \\
\hline $95 \%$ confidence interval $^{\mathrm{d}}$ & & $38.3-67.6$ & $38.3-67.9$ \\
\hline \multicolumn{4}{|l|}{ AE leading to death } \\
\hline No. of patients with $\mathrm{AE}$ & 0 & 0 & 0 \\
\hline No. of AEs & 0 & 0 & 0 \\
\hline Incidence $\%$ & 0 & 0 & 0 \\
\hline $95 \%$ confidence interval $^{c}$ & $0-7.3$ & $0-3.4$ & $0-3.2$ \\
\hline Difference (vs. Placebo) & & 0 & 0 \\
\hline $95 \%$ confidence interval $^{\mathrm{d}}$ & & - & - \\
\hline \multicolumn{4}{|l|}{ Serious AE excluding death } \\
\hline No. of patients with $\mathrm{AE}$ & 0 & 3 & 4 \\
\hline No. of AEs & 0 & 3 & 4 \\
\hline Incidence $\%$ & 0 & 2.8 & 3.5 \\
\hline $95 \%$ confidence interval $^{c}$ & $0-7.3$ & $0.6-8.0$ & $1.0-8.7$ \\
\hline Difference (vs. Placebo) & & 2.8 & 3.5 \\
\hline $95 \%$ confidence interval $^{\mathrm{d}}$ & & -5.1 to 8.1 & -4.3 to 8.9 \\
\hline \multicolumn{4}{|c|}{ AE leading to study drug discontinuation } \\
\hline No. of patients with $\mathrm{AE}$ & 0 & 13 & 14 \\
\hline No. of AEs & 0 & 16 & 17 \\
\hline Incidence \% & 0 & 12.1 & 12.3 \\
\hline $95 \%$ confidence interval $^{c}$ & $0-7.3$ & $6.6-19.9$ & $6.9-19.7$ \\
\hline Difference (vs. Placebo) & & 12.1 & 12.3 \\
\hline $95 \%$ confidence interval $^{\mathrm{d}}$ & & $2.1-19.9$ & $3.2-19.8$ \\
\hline \multicolumn{4}{|l|}{ AE without residual/AEs } \\
\hline No. of AEs without residual & 29 & 240 & 255 \\
\hline Incidence $(\%)$ & 90.6 & 94.5 & 94.4 \\
\hline
\end{tabular}


Table 3 continued

\begin{tabular}{|c|c|c|c|}
\hline Adverse event (AE) & $\begin{array}{l}\text { Placebo } \\
(N=49)\end{array}$ & $\begin{array}{l}\text { Favipiravir } \\
(N=107)^{\mathrm{a}}\end{array}$ & $\begin{array}{l}\text { Pooled favipiravir } \\
(N=114)^{\mathbf{b}}\end{array}$ \\
\hline $95 \%$ confidence interval $^{c}$ & $75.0-98.0$ & $90.9-97.0$ & $91.0-96.9$ \\
\hline Difference (vs. Placebo) & & 3.9 & 3.8 \\
\hline $95 \%$ confidence interval $^{\mathrm{d}}$ & & -4.1 to 20.4 & -4.1 to 20.5 \\
\hline \multicolumn{4}{|l|}{ Untreated $\mathrm{AE} / \mathrm{AEs} \mathrm{s}^{\mathrm{e}}$} \\
\hline No. of untreated AEs & 10 & 167 & 177 \\
\hline Incidence (\%) & 31.3 & 65.7 & 65.6 \\
\hline $95 \%$ confidence interval $^{c}$ & $16.1-50.0$ & $59.6-71.6$ & $59.6-71.2$ \\
\hline Difference (vs. Placebo) & & 34.5 & 34.3 \\
\hline $95 \%$ confidence interval $^{d}$ & & $13.7-50.2$ & $13.7-49.9$ \\
\hline
\end{tabular}

Values are through Day 28, the date of the scheduled completion of the trial intervention

a All patients who were assigned to favipiravir treatment and administered favipiravir at least once

b All patients who were administered favipiravir at least once, including those who were switched to favipiravir treatment for rescue

c Confidence intervals were calculated by the Clopper-Pearson method

d Confidence intervals for the differences were calculated by the test-based exact method

e Adverse events that did not require intervention

however, the time to improvement when these two parameters were combined was 1.6 days shorter in the favipiravir group. These results suggested that improvement in body temperature and improvement in respiratory status did not correlate. The composite outcome as the primary endpoint was considered to be a sensitive endpoint for patients with COVID-19 showing such non-uniform symptoms transition. One possible reason for the lack of differences in these two clinical parameters was the acceptance of concomitant use of corticosteroids. In a subgroup analysis of patients with and without corticosteroids, the latter showed approximately twice as high HRs as the former, indicating that favipiravir treatment appeared to be preferable. Another possible reason was that approximately $20 \%$ of patients maintained $96 \%$ or higher $\mathrm{SpO}_{2}$ throughout the study and did not deteriorate. The presence of these patients may have reduced the difference between the two groups.
The HR for the time to recovery to SARSCoV-2-negative, which showed a significant difference by a log-rank test, was approximately 1.40. This HR was comparable to studies in which favipiravir was administered to asymptomatic or mild COVID-19 patients in Japan [15] or in a relatively similar patient population in India [13], although there were no significant differences in viral clearance in either trial. In addition, an interim report on the favipiravir trial in Russia in patients with moderate COVID-19 also suggested early viral clearance [14]. Therefore, it was considered that these results indicated a consistent degree of antiviral activity of favipiravir against SARS-CoV-2.

In a hamster SARS-CoV-2 infection model, the infectious virus titer fell significantly and lung tissue improved considerably after favipiravir administration at plasma concentrations $\geq$ $4.4 \pm 1.6 \mu \mathrm{g} / \mathrm{mL}$ [16]. Unfortunately, there are no pharmacokinetic data in the present trial, but the trough concentrations were approximately ten-fold higher than the 
aforementioned hamster plasma concentrations when the same favipiravir dose as in the present trial was administered to patients with SFTS [10]. In addition, favipiravir has been presumed to have good penetration into the lungs [17]. These findings were consistent with that reported in the present trial, in which the difference in improvement rates on chest imaging between the two groups gradually became more apparent, with the differences becoming significant from Day 10 and beyond. Although the viral dynamics within hamster lungs cannot be directly compared to those of human patients, the favipiravir-induced reduction in viral load in the lungs could have been responsible for the difference in improvement rates in chest imaging findings.

In the evaluation based on the 7-point ordinal scale, there was no significant difference in the time course of clinical status. As the reason for this, the number of patients with deterioration in the placebo group may have been reduced by patients who were judged as having a lack of efficacy in the placebo group and being switched to favipiravir treatment, which could have biased in the direction of no difference.

From the results of subgroup analysis, the HRs of the primary endpoint were above 2.0 in patients with risk factors of severe illness, such as obesity or diabetes mellitus, as well as in patients with early-onset, which indicated that use of favipiravir would be desirable in patients with these characteristics. Fujii et al. [18] reported that early administration of favipiravir shortened the time to afebrile state in a retrospective cohort study. We were able to confirm that early favipiravir intervention led to more favorable clinical outcomes in moderate COVID-19 patients by a randomized, placebocontrolled trial. Given the mechanism of action of favipiravir acting on the viral replication process, this finding would be reasonable.

The incidence of AEs in the favipiravir group was significantly higher than in the placebo group. However, the vast majority of AEs in the favipiravir patients were transient, and the incidence of AEs that disappeared without intervention or improved was much higher in the favipiravir group. AEs documented in the favipiravir group were predominantly related to hyperuricemia. A previous trial conducted on asymptomatic or mild Japanese patients with COVID-19 also observed 69 (84.1\%) of 82 patients given favipiravir experienced transient hyperuricemia [15]. Thus, transient uric acidrelated AEs involving favipiravir use were common, and the vast majority of them were subclinical. On the other hand, favipiravir was administered to 10,986 patients with COVID-19 in a Japanese observational study, and the development of gout was reported in $14(0.1 \%)$ patients [8]. While further investigations are needed, administration of favipiravir should be considered with judiciousness in patients with a history of hyperuricemia or gout. Two SAEs were reported to have a potentially causal relationship with favipiravir. Of these, cerebral infarction could have been the result of a thrombotic complication associated with COVID-19 [19, 20].

This trial has a number of limitations that should be addressed. First, there was an inherent operational bias due to the single-blind design. To minimize the effects of this bias, the primary endpoint was re-assessed under blind control by an independent Central Committee. The second limitation was that virological investigations were measured solely by nasopharyngeal swabs, despite targeting COVID-19 patients with pneumonia. Ideally, the trial should have evaluated viral dynamics in the lower respiratory tract; however, the collection process would have been an additional burden to medical professionals dealing with this pandemic. Third, the trial encountered difficulty in recruiting only suitable patients of early-onset for evaluating antiviral drug efficacy. Therefore, the results may have underestimated the effects of antiviral therapeutics. In view of the viral dynamics described in the report [21], future studies should recruit patients who are at least within 4 days of onset. Fourth, the present results are based on COVID19 patients with moderate pneumonia $\left(\mathrm{SpO}_{2} \geq 94 \%\right)$, and, thus, cannot be applied to patients with $\mathrm{SpO}_{2} \leq 93 \%$ or more severe illness. Finally, the primary endpoint was set based on the COVID-19 patient discharge criterion at that time, and cannot be directly applied to the current criterion. 
Recently, SARS-CoV-2 mutations have been reported worldwide and the emergence of viral mutations could have implications for the development of treatments and vaccines [22-24]. Favipiravir is not a drug that acts on a spike-protein of SARS-CoV-2, which is often mutated, so it is assumed that it is not so affected by viral mutations. Such characteristics of favipiravir may be important as treatment options for COVID-19 in the future.

\section{CONCLUSIONS}

Favipiravir appeared to shorten the time until clinical improvement by approximately 3 days in patients with moderate COVID-19 pneumonia presenting with $\mathrm{SpO}_{2} \geq 94 \%$ as per the analysis plan. The result was mostly in line with the results in previous unblinded trials. Of the individual COVID-19 signs and symptoms, chest imaging, viral clearance, headache, myalgia or arthralgia, and fatigue or tiredness improved significantly earlier with favipiravir treatment. Furthermore, favipiravir showed significantly higher HRs in patients with earlyonset or known risk factors for severe illness. At the same time, it should be noted that favipiravir treatment resulted in a significantly higher incidence of AEs. Although some limitations exist in this trial, the result indicates that favipiravir might be one of the options for moderate COVID-19 pneumonia treatment. The risk of AEs, including hyperuricemia, should be carefully considered when using it.

\section{ACKNOWLEDGEMENTS}

The authors thank the patients for contributing to this work. The authors are also grateful to everyone who bravely treated the COVID-19 patients, to Taiichiro Kobayashi M.D. Ph.D. (Tokyo Metropolitan Cancer and Infectious Diseases Center Komagome Hospital, Tokyo, Japan) who made a great contribution to patient recruitment, to Ms. Kiyomi Kabasawa (Tokyo Shinagawa Hospital, Tokyo, Japan), Ms. Kaoru Sato (I'rom EC Co., Ltd., Tokyo, Japan) who assisted with data entry, and to all other
Clinical Research Coordinators involved in patient enrollment at the study centers. The authors are also grateful to the Central Committee members for their independent evaluation of the study: Yasuyuki Kato, M.D., M.P.H (International University of Health and Welfare, School of Medicine, Narita, Chiba), Hideki Araoka, M.D. (Toranomon Hospital, Tokyo, Japan), Ichiyo Obara, M.D. Ph.D. (Japan SelfDefense Forces Yokosuka Hospital, Kanagawa, Japan), Shigeki Misumi, M.D. Ph.D. (Jikei University School of Medicine, Tokyo, Japan), and Toshihito Furukawa (Biostatistical Research Co., Ltd., Tokyo, Japan).

Funding. This trial was sponsored by FUJIFILM Toyama Chemical Co., Ltd. The Rapid Service Fees were funded by the sponsor.

Authorship. All named authors meet the International Committee of Medical Journal Editors (ICMJE) criteria for authorship for this article, take responsibility for the integrity of the work as a whole, and have given their approval for this version to be published.

Authorship Contributions. Conceptualization: Satoshi Iwata, Tsutomu Sakurai; Methodology: Satoshi Iwata, Osamu Kobayashi, Hiroyuki Kunishima, Masaharu Shinkai; Formal analysis: Yoshihiko Matsumoto, Tsutomu Sakurai; Investigation: Masaharu Shinkai, Kenji Tsushima, Shingo Tanaka, Eri Hagiwara, Norihito Tarumoto, Ichiro Kawada, Yuji Hirai, Sho Fujiwara, Yuko Komase, Takeshi Saraya, Hidefumi Koh, Naho Kagiyama, Megumi Shimada, Daiki Kanou, Shinichi Antoku, Yujiro Uchida, Yutaka Tokue, Mikio Takamori, Yasuhiro Gon, Kenya Ie, Yoshitaka Yamazaki, Kazumasa Harada, Naoki Miyao, Takashi Naka, Mitsunaga Iwata, Atsushi Nakagawa, Kazutoshi Hiyama, Yoshihiko Ogawa, Masahiro Shinoda, Shinichiro Ota, Takatomo Hirouchi, Jiro Terada, Shuichi Kawano and Takashi Ogura; Supervision: Satoshi Iwata; Writing - original draft preparation; Masaharu Shinkai. All authors commented on previous versions of the manuscript. All authors read and approved the submitted version. 
Disclosures. Tsutomu Sakurai and Yoshihiko Matsumoto are employees of the sponsor. Masaharu Shinkai, Kenji Tsushima, Shingo Tanaka, Eri Hagiwara, Norihito Tarumoto, Ichiro Kawada, Yuji Hirai, Sho Fujiwara, Yuko Komase, Takeshi Saraya, Hidefumi Koh, Naho Kagiyama, Megumi Shimada, Daiki Kanou, Shinichi Antoku, Yujiro Uchida, Yutaka Tokue, Mikio Takamori, Yasuhiro Gon, Kenya Ie, Yoshitaka Yamazaki, Kazumasa Harada, Naoki Miyao, Takashi Naka, Mitsunaga Iwata, Atsushi Nakagawa, Kazutoshi Hiyama, Yoshihiko Ogawa, Masahiro Shinoda, Shinichiro Ota, Takatomo Hirouchi, Jiro Terada, Shuichi Kawano, Takashi Ogura, Hiroyuki Kunishima, Osamu Kobayashi and Satoshi Iwata have nothing to disclose. Sho Fujiwara is currently affiliated with Department of Emergency Medicine, Tokyo Hikifune Hospital, 2-27-1 HigashiMukojima, Sumida-ku, Tokyo 131-0032, Japan. Daiki Kanou is currently affiliated with Department of Respiratory Medicine, Yokohama City University Hospital, 3-9 Fukuura, Kanazawa-ku, Yokohama, Kanagawa 236-004, Japan.

Compliance with Ethics Guidelines. This trial plan was filed to Japanese regulatory authorities (IND \#2019-7269). The protocol, informed consent form, and all other required documents were reviewed by an Institutional Review Board (IRB) of each trial site prior to initiation of the trial at that site. The trial was approved by all IRBs (please see supplementary material) and was conducted in accordance with the Helsinki Declaration (1964 and its later amendments) and Good Clinical Practice.

Data Availability. The datasets generated during and/or analyzed during the current study are currently being submitted to regulatory authorities due to NDA and are not publicly available. The datasets will be available from the sponsor on reasonable request after regulatory approval.

Open Access. This article is licensed under a Creative Commons Attribution-NonCommercial 4.0 International License, which permits any non-commercial use, sharing, adaptation, distribution and reproduction in any medium or format, as long as you give appropriate credit to the original author(s) and the source, provide a link to the Creative Commons licence, and indicate if changes were made. The images or other third party material in this article are included in the article's Creative Commons licence, unless indicated otherwise in a credit line to the material. If material is not included in the article's Creative Commons licence and your intended use is not permitted by statutory regulation or exceeds the permitted use, you will need to obtain permission directly from the copyright holder. To view a copy of this licence, visit http://creativecommons.org/licenses/by$\mathrm{nc} / 4.0 /$.

\section{REFERENCES}

1. Coronavirus disease (COVID-19) Weekly Epidemiological Update and Weekly Operational Upd. Geneva: World Health Organization. 2021. https:// www.who.int/publications/m/item/weeklyoperational-update-on-covid-19-10-may-2021. Accessed 10 May 2021.

2. Gowen $\mathrm{BB}$, Wong $\mathrm{MH}$, Jung $\mathrm{KH}$, et al. In vitro and in vivo activities of T-705 against arenavirus and bunyavirus infections. Antimicrob Agents Chemother. 2007;51:3168-76.

3. Mendenhall M, Russell A, Smee DF, et al. Effective oral favipiravir (T-705) therapy initiated after the onset of clinical disease in a model of arenavirus hemorrhagic fever. PLoS Negl Trop Dis. 2011;5: e1342.

4. Wang M, Cao R, Zhang L, et al. Remdesivir and chloroquine effectively inhibit the recently emerged novel coronavirus (2019-nCoV) in vitro. Cell Res. 2020;30:269-71.

5. Shanon A, Selisko B, Le NT, et al. Rapid incorporation of favipiravir by the fast and permissive viral RNA polymerase complex results in SARS-CoV-2 lethal mutagenesis. Nat Commun. 2020;11:4682.

6. Gordon CJ, Tchesnokov EP, Woolner E, et al. Remdesivir is a direct-acting antiviral that inhibits RNA-dependent RNA polymerase from severe acute respiratory syndrome coronavirus 2 with high potency. J Biol Chem. 2020;295:6785-97.

7. Cai Q, Yang M, Liu D, et al. Experimental treatment with favipiravir for COVID-19: An open-label control study. Eng (Beijing). 2020;6:1192-8. 
8. Favipiravir Observational Study Group, Fujita Health University. Interim report of the Favipiravir Observational Study in Japan. 2020. https://www. kansensho.or.jp/uploads/files/topics/2019ncov/ covid19_favip_210419_eng.pdf.

9. Sidwell RW, Barnard DL, Day CW, et al. Efficacy of orally administered T-705 on lethal avian influenza A (H5N1) virus infections in mice. Antimicrob Agents Chemother. 2007;51:845-51.

10. Suemori K, Saijo M, Yamanaka A, et al. A multicenter non-randomized, uncontrolled single arm trial for evaluation of the efficacy and the safety of the treatment with favipiravir for patients with severe fever with thrombocytopenia syndrome. PLoS Negl Trop Dis. 2021;22:e0009103.

11. Cui L, Hung HM, Wang SJ. Modification of sample size in group sequential clinical trials. Biometrics. 1999;55:853-7.

12. Morikawa M, Shinoda M, Ota S, et al. Clinical features of 154 COVID-19 patients and parameters for effective detection of pneumonia at the time of initial diagnosis in Japan. Intern Med. 2021;60: 31-7.

13. Udwadia ZF, Singh P, Barkate H, et al. Efficacy and safety of favipiravir, an oral RNA-dependent RNA polymerase inhibitor, in mild-to-moderate COVID19: a randomized, comparative, open-label, multicenter, phase 3 clinical trial. Int $\mathrm{J}$ Infect Dis. 2021;103:62-71.

14. Ivashchenko AA, Dmitriev KA, Vostokova NV, et al. AVIFAVIR for treatment of patients with moderate COVID-19: interim results of a phase II/III multicenter randomized clinical trial. Clin Infect Dis. 2020;2020:ciaa1176.

15. Doi $Y$, Hibino M, Hase R, et al. A prospective, randomized, open-label trial of early versus late favipiravir therapy in hospitalized patients with
COVID-19. Antimicrob Agents Chemother. 2020;64:e01897-20.

16. Kaptein SJF, Jacobs S, Langendries L, et al. Favipiravir at high doses has potent antiviral activity in SARS-CoV-2-infected hamsters, whereas hydroxychloroquine lacks activity. Proc Natl Acad Sci USA. 2020;117:26955-65.

17. Wang Y, Chen L. Tissue distributions of antiviral drugs affect their capabilities of reducing viral loads in COVID-19 treatment. Eur J Pharmacol. 2020;889: 173634.

18. Fujii S, Ibe Y, Ishigo T, et al. Early favipiravir treatment was associated with early defervescence in non-severe COVID-19 patients. J Infect Chemother. 2021;27:1051-7.

19. Zhou F, Yu T, Du R, et al. Clinical course and risk factors for mortality of adult inpatients with COVID-19 in Wuhan, China: a retrospective cohort study. Lancet. 2020;395:1054-62.

20. Oxley TJ, Mocco J, Majidi S, et al. Large-vessel stroke as a presenting feature of Covid-19 in the young. N Engl J Med. 2020;382:e60.

21. Wölfel R, Corman VM, Guggemos W, et al. Virological assessment of hospitalized patients with COVID-2019. Nature. 2020;581:465-9.

22. Galloway SE, Paul P, MacCannell DR, et al. Emergence of SARS-CoV-2 B.1.1.7 Lineage-United States, December 29, 2020-January 12, 2021. MMWR Morb Mortal Wkly Rep. 2021;70:95-9.

23. Li Q, Wu J, Nie J, et al. The impact of mutations in SARS-CoV-2 spike on viral infectivity and antigenicity. Cell. 2020;182:1284-94.

24. Cele S, Gazy I, Jackson L, et al. Escape of SARS-CoV2 501Y.V2 from neutralization by convalescent plasma. Nature. 2021;593:142-6. 